\title{
Pedestrian Flow Characteristics Under Heterogeneous Traffic Conditions
}

\author{
A. M. Tahsin Emtenan*, Showkat Ibne Shahid \\ Department of Civil Engineering, Bangladesh University of Engineering and Technology, Dhaka, Bangladesh
}

Email address:

emtenan.buet@gmail.com (A. M. T. Emtenan), showkat_shahid@yahoo.com (S. I. Shahid)

${ }^{*}$ Corresponding author

\section{To cite this article:}

A. M. Tahsin Emtenan, Showkat Ibne Shahid. Pedestrian Flow Characteristics Under Heterogeneous Traffic Conditions. American Journal of Civil Engineering. Vol. 5, No. 5, 2017, pp. 282-292. doi: 10.11648/j.ajce.20170505.13

Received: July 3, 2017; Accepted: August 16, 2017; Published: August 28, 2017

\begin{abstract}
Pedestrians are an important element of the traffic stream especially in a country like Bangladesh. Dhaka is the biggest metropolitan city of Bangladesh and thus people from different parts of the country are relocating to Dhaka. This has rendered Dhaka one of the most densely populated cities in the World. With the resource restraint and crumbling infrastructure, the transportation facilities can't cope up with the ever growing population. A major proportion of the population are the urban slum dwellers and low income people who are left with the only option of walking. Pedestrians are the most vulnerable roadway users. Ironically they are not given due consideration in the design of roadway features. However, the alarming growths of pedestrians, inadequate sidewalks and their haphazard movement have forced them to share the carriageway with the vehicles. This has led to a shocking number of accidents in the past as well as contributed to further increasing the congestion on the carriageway. This study has attempted to present flow characteristics of pedestrians on exclusive sidewalks in three important urban areas of Dhaka namely Farmgate, Shahbag and Shukrabad. Pedestrian flow, density and walking speed are considered as the three primary parameters on which the flow characteristics depend. These are essential tools for determining the sidewalk capacity which will help in sidewalk design improvements required. The relationships between the three parameters were expressed in terms of three equations which makes it easy to comprehend the situations in these three locations. The level of service (LOS) has been determined at each location also. On the basis of flow rate, Farmgate has a LOS $\mathrm{C}$ and Shukrabad and Shahbag both have a LOS B. The average free-flow speed from three locations is about $1.18 \mathrm{~m} / \mathrm{s}$. This might help create an idea about the rate at which pedestrians might clear out from facilities.
\end{abstract}

Keywords: Speed, Flow, Density, Pedestrian Trap, Level of Service

\section{Introduction}

Bangladesh, a country in South Asia is very densely populated with about 150 million inhabitants living in an area of 147570 sq. km. Despite rising incomes and GDP, the distribution of wealth remains skewed in this country. Dhaka itself is dominated by people living under the poverty line. For this portion of the population, walking is the primary choice for making trips. Yet pedestrians have received far less attention than vehicular traffic. Pedestrians are the most vulnerable and neglected road users in Bangladesh. Every person is a pedestrian at some point in a day as all journeys necessarily start or end as a walking trip. Walking is directly involved with using other modes. The importance of pedestrian movements is understood globally and cannot be overemphasized. However, the roads of Dhaka Metropolitan City are threatening to pedestrians. Hence, they require preferential treatment and exclusive facilities for their movement [1].

At present nearly 60 percent of urban trips involved walking alone in Dhaka city and it is particularly prevalent for short trips (DITS) [5]. In terms of road usage, at some locations, pedestrians accounted for the highest number, representing nearly 62 percent of the total user groups in Dhaka, the capital of Bangladesh. Low motorization levels, unplanned haphazard land use, road side industry, inadequate pedestrian facility and the severe lack of priority and attention given to pedestrians in the traditional transport planning and traffic management cause serious hazardous 
situations. Since walking is a major contributor to a sustainable transport strategy, it requires special attention. Yet pedestrian can still claim to be the most forgotten and neglected user group. Pedestrian do not need a license to use the roads, they are a mobile group and are generally able to go almost anywhere. Pedestrians are dispersed across the road network and can be seen all time, day and night, in all weathers, and on all types of roads [6]. Therefore, they are very important and need special consideration during design of roadway features. If exclusive facilities are not provided for the pedestrians, then the friction would increase with the development of sides, provision of on-street parking, and movement of the vehicles. At times, shoulders are provided on both the sides of road, which can also be used by the pedestrians [7]. But many times these shoulders are used by the vehicles for parking or are encroached by venders or hawkers and shopkeepers. In such cases, even with the provision of side shoulders, the pedestrians have no option but to walk on the carriageway along the vehicular traffic. It increases the conflict between the pedestrians and the vehicles. Such conditions are very common on urban roads of medium-sized cities in Bangladesh and many other developing nations. It is therefore important to study the characteristics of the pedestrian under mixed traffic condition and to compare them with those reported from other countries. Overall there are wide differences between developed and developing countries in the behavior, knowledge and attitudes of the pedestrians and the preferential treatment they deserve. Their logic and technology may be adjusted to match with the condition of our country. In Bangladesh, little attention has been devoted to study pedestrian behavior and pedestrian flow characteristics [2].

This paper aims at establishing relationships among primary flow parameters namely flow, density and speed of the pedestrians in three major urban areas of Dhaka Metropolitan City. The study was conducted and data were collected in such a way that flow conditions were not affected by the procedure itself. The specific objectives of the study are:

1. To obtain flow fluctuation patterns during peak and offpeak hours in three major areas of Dhaka Metropolitan City.

2. To establish the primary mathematical relationships between speed-density, speed-flow and flow-density.

3. To estimate the free flow speed obtained from the equations derived.

4. To determine the maximum pedestrian flow in each site.

5. To determine the level of service (LOS) for three sites based on free flow speed as well as maximum flow rate.

6. To compare the flow characteristics parameters as well as LOS within different sites.

\section{Pedestrian Flow Characteristics}

General relationships used to relate the parameters of pedestrian flow characteristics are asfollows:

Pedestrian walking speed $(\mu)$ and density $(\mathrm{k})$

$$
\mu=\mathrm{a}-\mathrm{b} * \mathrm{k}
$$

Pedestrian flow (q) and density (k)

$$
\mathrm{q}=\mathrm{a} * \mathrm{k}-\mathrm{b} * \mathrm{k}^{2}
$$

Pedestrian walking speed $(\mu)$ and flow (q)

$$
\mathrm{Q}=\mu * \mathrm{k}=\mu * \frac{(a-\mu)}{b}
$$

Here ' $a$ ' and ' $b$ ', denote the model parameters which vary from location to location. In this study, single regime approach will be used for establishing relationships between the three basic parameters of pedestrian flow. That means the model parameters found from speed density relation would be used to determine flow-density and flow-speed relations (Rastogi et. al. 2008). The values of pedestrian flow, density and walking speeds were inserted in Excel sheets with corresponding time. The speed density columns were used to draw a speed vs. density best fit linear graph. This was done for each location. The actual data points around the best fit line shows the distribution of the data around the line. The values of model parameters ' $a$ ' and 'b'were used to formulate the flow-density and speed-flow equations [3].

\section{Methodology}

\subsection{Preliminary Survey and Site Selection}

Before the onset of the study preliminary surveys were conducted in different major areas of Dhaka Metropolitan City to identify the study areas. The study is based on the pedestrian movement characteristics, hence the surveys focused on the pedestrians only [4]. The criterions considered for selecting study areas include:

1. Exclusive sidewalk for the pedestrians.

2. Distinctive flow fluctuation throughout the day. That is some period of the day must have low pedestrian flow and density, while other hours of the day might have heavy congestion of pedestrians.

3. Friction caused by street hawkers/vendors or construction works should be minimum.

4. A suitable elevated space must be present for collection of data, suitable an elevated pedestrian overpass.

The preliminary surveys were conducted in Dhanmondi, Motijheel, Farmgate, Shahbaag areas. Based on the requirements of the study 3 locations were chosen; Farmgate, Shahbaag and Shukrabad of Dhanmondi area. Test videos were taken and data reduction feasibilities were checked. Pedestrian traps were chosen so that pedestrians are unaware of the trap while walking and hence, ensuring uninterrupted flow.

\subsection{Data Collection Schedule and Collection Period}

Data collection using video recording was done during typical weekends. 20 minutes of video were recorded, representing each hour of the day. For example, the data for 7:00-8:00 AM hour block were collected from 7:21 AM to 
7:40 AM which provides a fair representation of the entire hour of pedestrian movement. In this way for each site, ten sets of videos were recorded representing 10 hour blocks starting from 7:00 AM to 5:00 PM. Video recording was done in this way in order to capture the flow fluctuations occurring from 7:00 AM to 5:00 PM. Evening peak usually occur after 5:30 PM. But due to light insufficiency only the fluctuations during the day time was recorded. The video recorder can only capture up to 80 minutes of high quality footage at once. Hence the observations at each site were divided into 3 blocks of time period and data were collected on consecutive 3 weekdays assuming that flow conditions remain similar throughout the 3 days and the data collected was assigned for a single day.

\subsection{Data Reduction Procedures}

The video recorded pedestrian traffic data are now played to extract the required information. All the desired variables are obtained from playing back the videos. In this thesis we required 3 parameters of pedestrian traffic flow, namely flow, speed and density. Playing back the video yielded 200 sets of data for each location. The data were procured form the videos in the following method.

Flow Measurement: An imaginary line was considered along the mid-section of the pedestrian trap. The number of pedestrians who crossed this line in each minute from both directions was calculated. Since the flow of people in Farmgate was extremely high the number of people crossing the line from one direction was calculated at first. Then the video was played back to obtain the number of pedestrians from the other direction. The total pedestrian number was divided by the effective width of the sideway and then by the time period of observation to obtain pedestrian flow rate in the unit $\mathrm{ped} / \mathrm{m} / \mathrm{min}[10]$.

Density Measurement: The density for each minute was measured by pausing the video 3 times within that minute and counting the number of people on the pedestrian trap. The video was paused at 1st, 30th and 59th second of each minute. The average of these three densities provided a fair idea about pedestrian density. Then this average was divided by pedestrian trap are to obtain pedestrian density in the unit $\mathrm{ped} / \mathrm{m}^{2}$. In case of total absence of pedestrians at those particular seconds, data were collected for three different instances in each minute [11]. For example: If no pedestrians are present while the video was paused at 1 st second then pedestrian number within the first one third period of that minute was taken. Similarly, if no pedestrians were present at 30 th second, pedestrian count was taken within 21 st to 40 th second of that minute.

Speed Measurement: The only effective method for pedestrian walking speed in this method was to use space mean speed. At every minute 3 to 4 pedestrians are identified. The time taken for each pedestrian to cross the study trap was calculated. This was done by noting the time when the pedestrian entered the study trap and the time when the pedestrian left the study trap. The difference was then regarded as time taken for that pedestrian to cross the trap. The time taken by $3 / 4$ pedestrians are averaged to obtain the average time period required to traverse the trap. The trap length was then divided by that average period to obtain pedestrian walking speed. The average period was expressed to two digits after the decimal point. In the cases of Farmgate and Shahbaag the time taken by each pedestrian was calculated in integers. While the videos of Shukrabad area were played back by a video player which shows time in milliseconds.

\subsection{Pedestrian Flow Characteristics}

Before finding the mathematical equations the flow fluctuations at each location are shown with the help of graphs. The graphs are plotted for flow vs. time parameters in figures 1, 2 and 3. In order to plot the graphs, the average of 20 minutes of flow data for each hour block was taken. This was then expressed in the unit Ped/hour/m and bar charts were drawn to represent flow fluctuations. For Farmgate pedestrian trap the increase in flow occurs at 7:00 AM to 8:00 AM, 11:00 AM to 12:00 AM, 12:00 PM to 1:00 PM and 2:00 PM to 3:00 PM. Shahbag experiences spike in pedestrian flow from 8:00 AM to 9:00 AM and 11:00 AM to 2:00 PM. Shukrabad also experiences a spike in flow from 2:00 PM to 4:00 PM. The probable causes are evaluated at the 'Discussion of Results' portion of the paper.

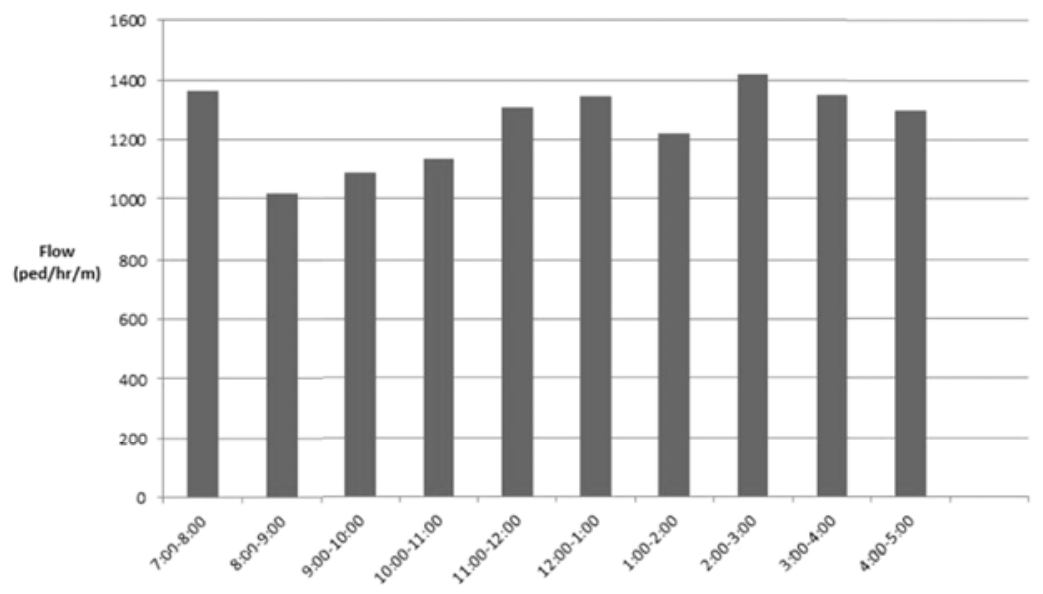

Figure 1. Flow fluctuation at Farmgate. 


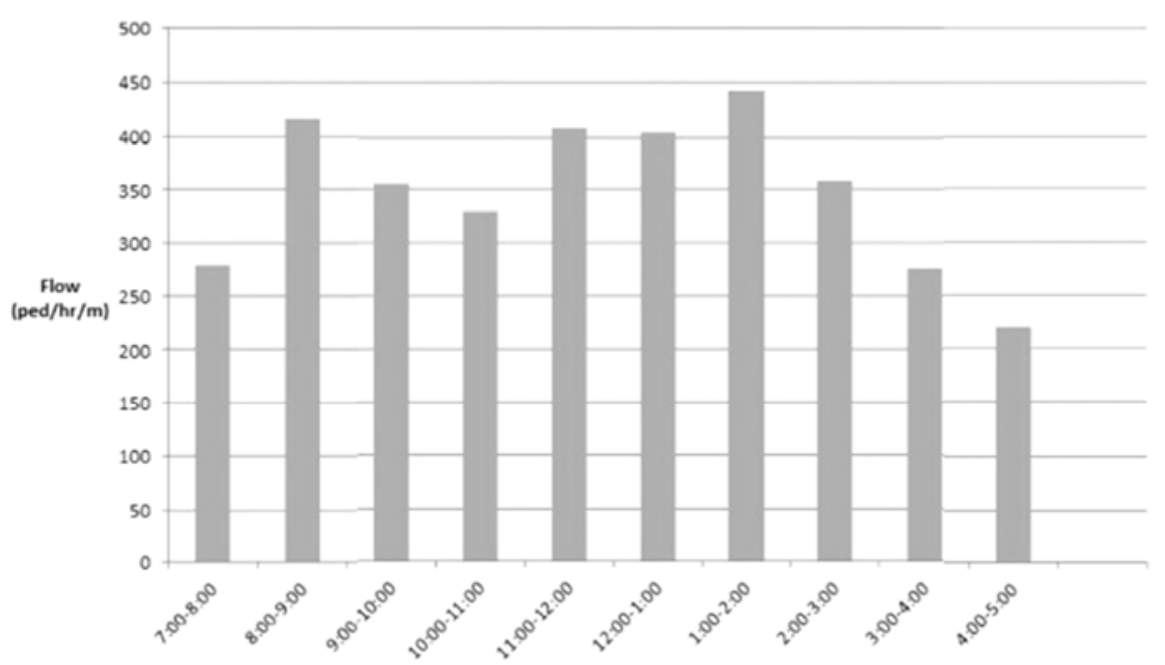

Figure 2. Flow fluctuation at Shahbag.

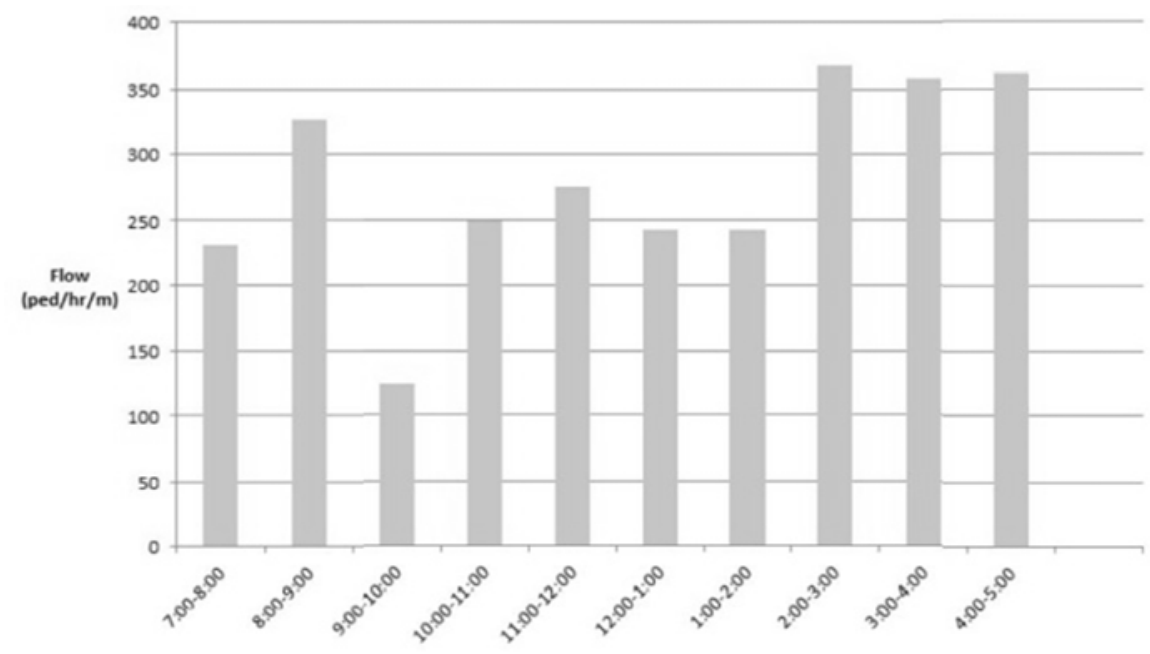

Figure 3. Flow fluctuation at Shukrabad.

\subsubsection{Speed-Density Relationship}

Linear regression of speed-density data is done for each location. The findings are discussed in this subsection.

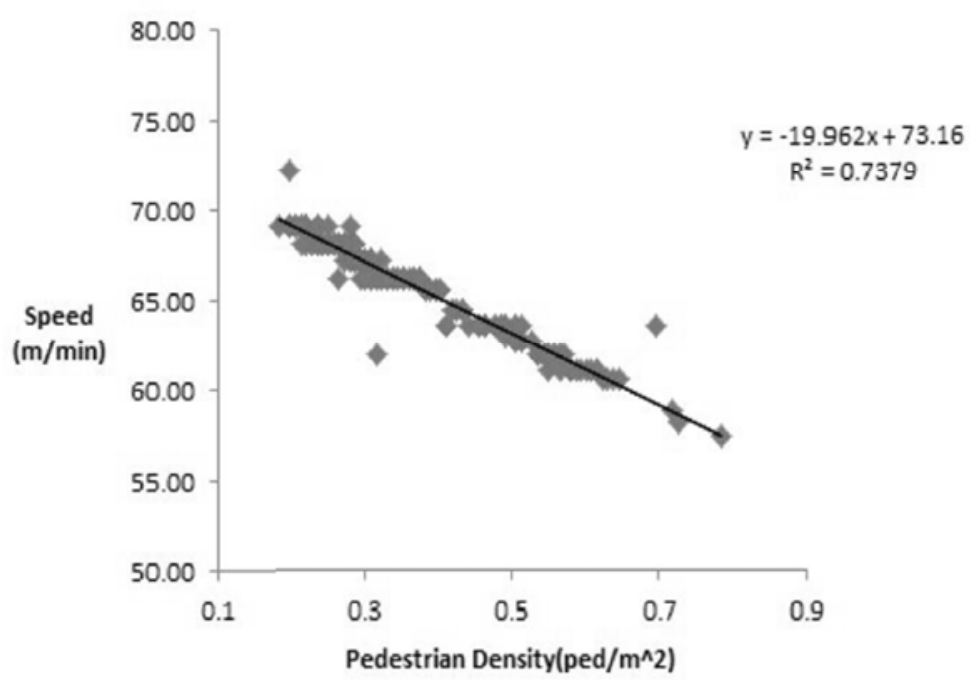

Figure 3. Pedestrian Speed-density curve at Farmgate. 
The least square linear regression between speed and density provides model parameters $a=69.49$ and $b=30.77$.

Therefore, fundamental equations of pedestrian flow provides the relations,

$$
\mu=73.16-19.96 \mathrm{k}
$$

$$
\begin{gathered}
\mathrm{q}=73.16 \mathrm{k}-19.96 \mathrm{k}^{2} \\
\mathrm{q}=\frac{\mu(73.16-\mu)}{19.96} \\
\mathrm{R}^{2}=0.74
\end{gathered}
$$

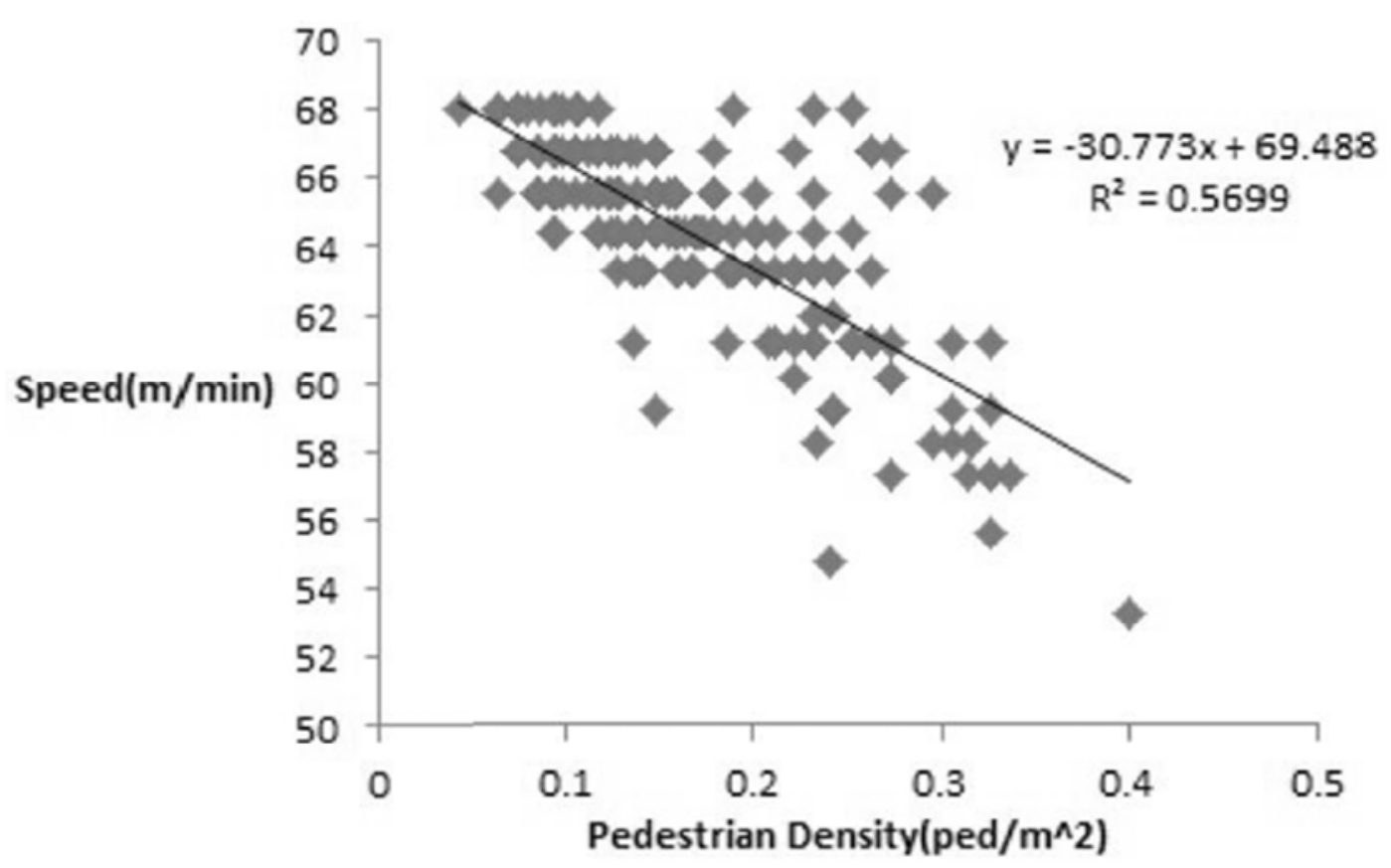

Figure 4. Pedestrian Speed-density curve at Shahbag.

The least square linear regression between speed and density provides model parameters $a=69.49$ and $b=30.77$.

Therefore, fundamental equations of pedestrian flow provides the relations,

$$
\mu=69.49-30.77 \mathrm{k}
$$

$$
\begin{gathered}
\mathrm{q}=69.49 k-30.77 \mathrm{k}^{2} \\
\mathrm{q}=\frac{\mu(69.48-\mu)}{30.77} \\
\mathrm{R}^{2}=0.57
\end{gathered}
$$

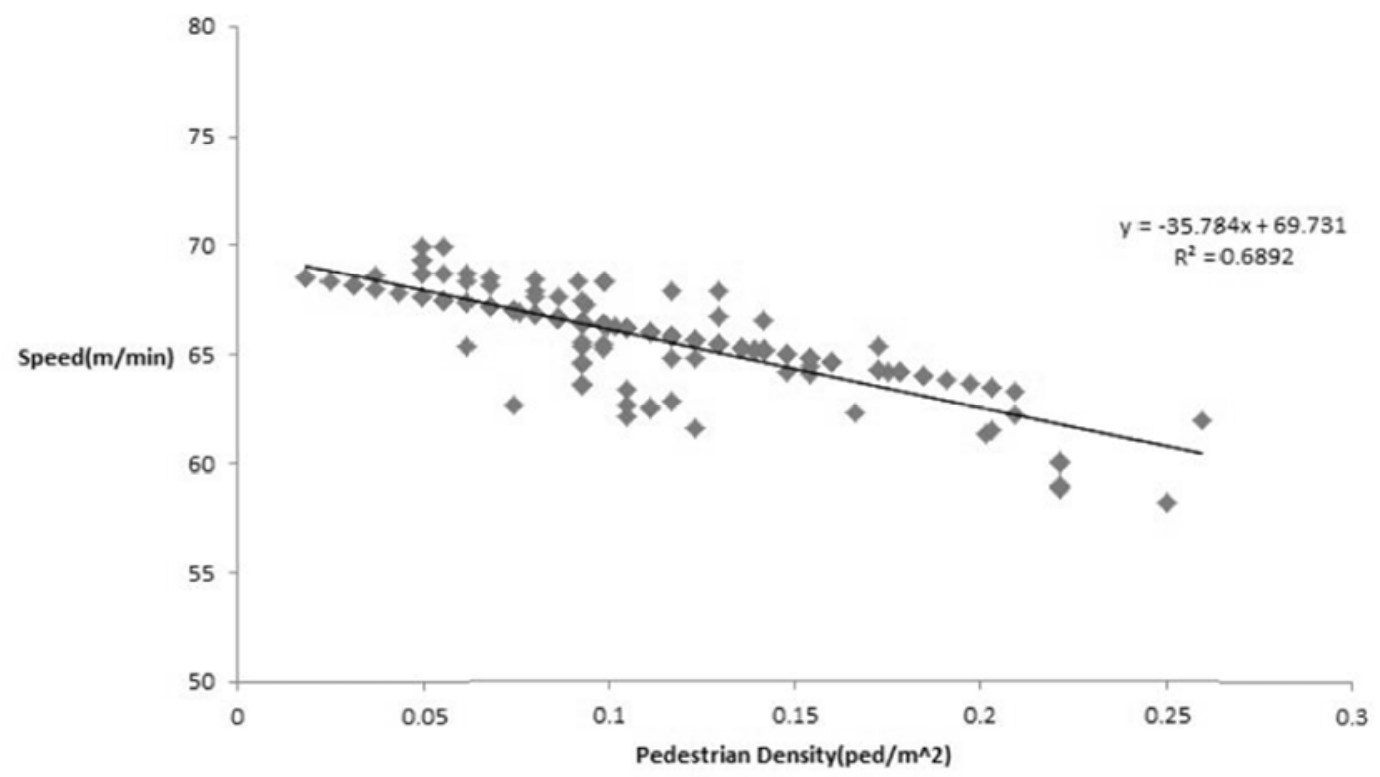

Figure 5. Pedestrian Speed-density curve at Shukrabad. 
The least square linear regression between speed and density provides model parameters $a=69.49$ and $b=30.77$.

Therefore, fundamental equations of pedestrian flow provides the relations,

$$
\begin{gathered}
\mu=69.73-35.78 \mathrm{k} \\
\mathrm{q}=69.73 \mathrm{k}-35.78 \mathrm{k}^{2} \\
\mathrm{q}=\frac{\mu(69.73-\mu)}{35.78} \\
\mathrm{R}^{2}=0.69
\end{gathered}
$$

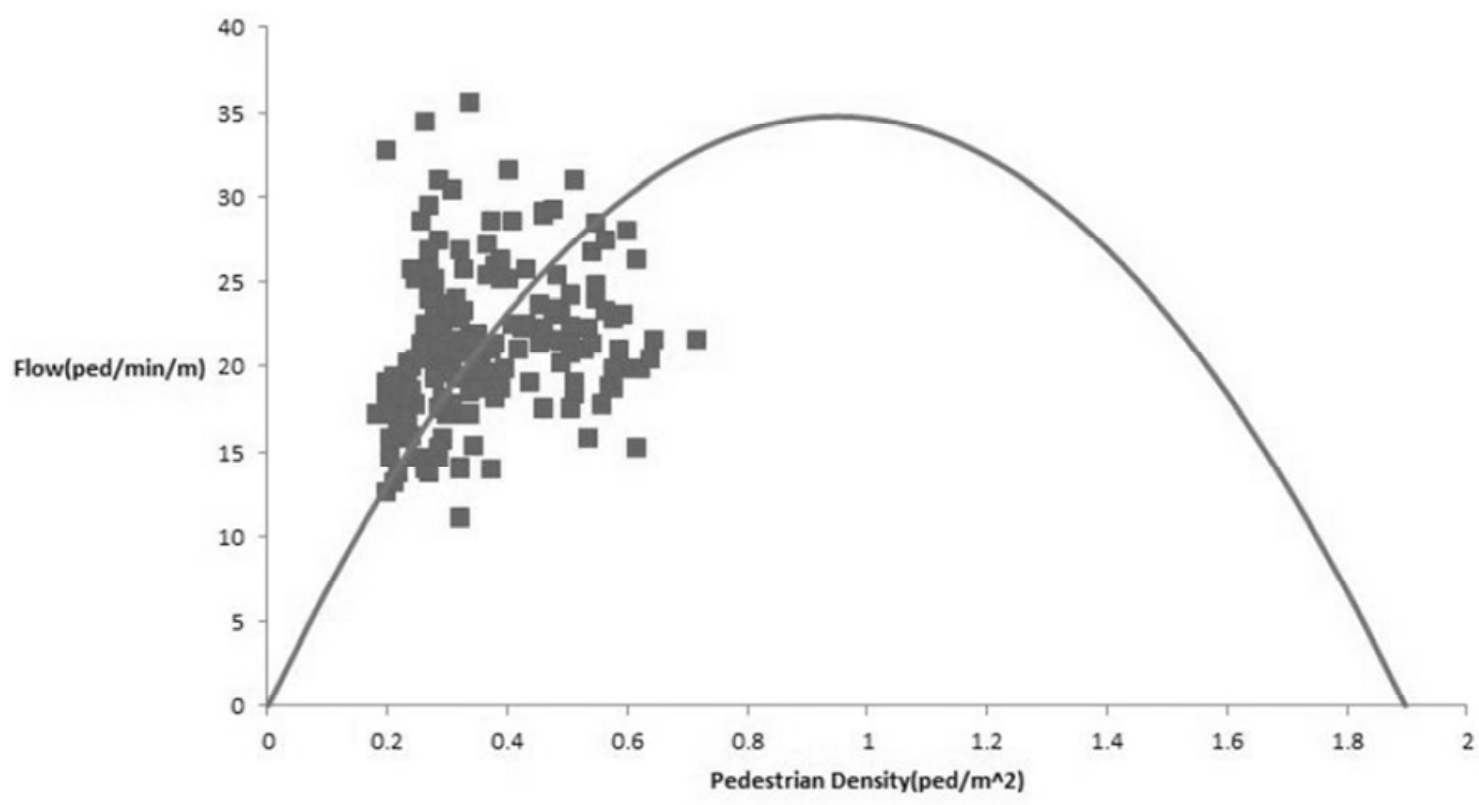

Figure 7. Flow vs. density at Farmgate.

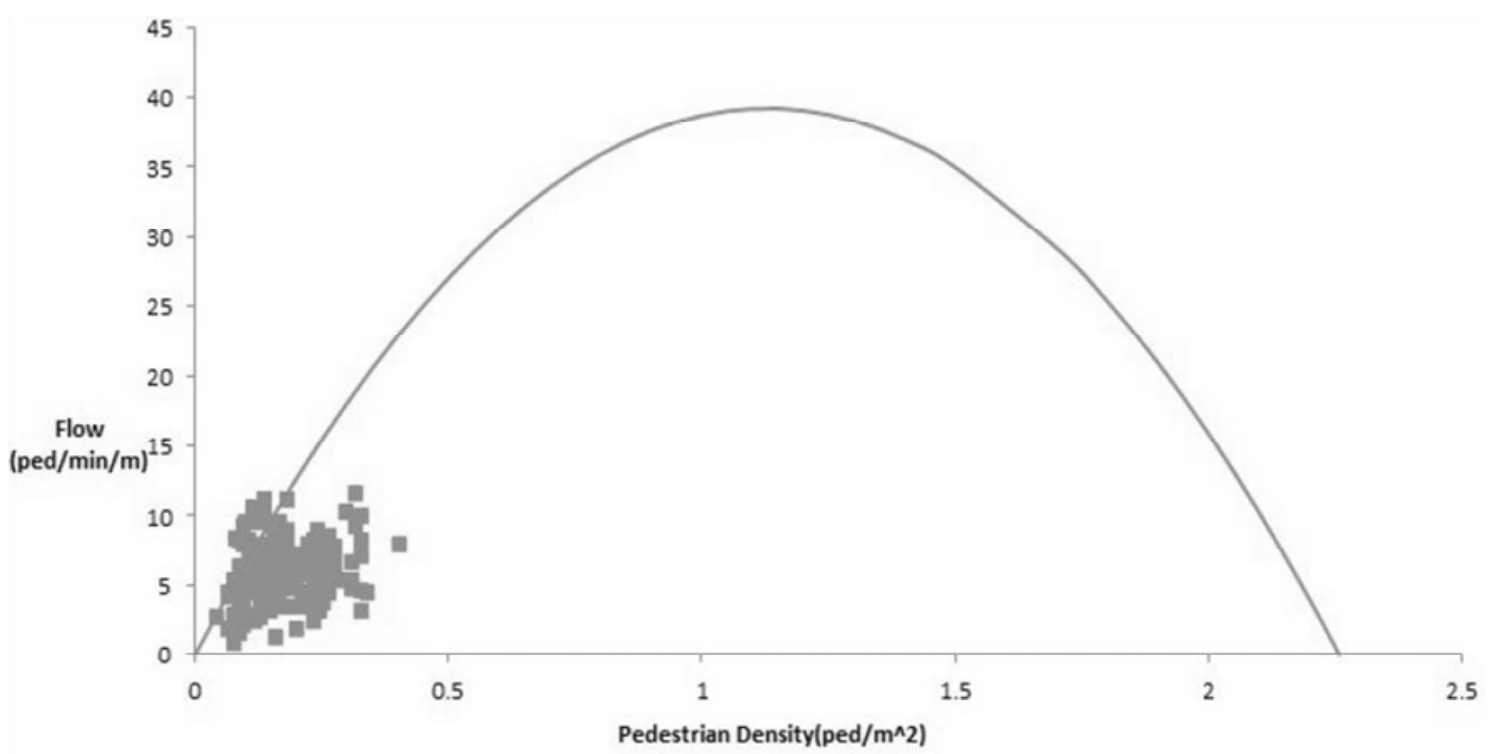

Figure 6. Flow vs. density at Shahbag.

\subsubsection{Flow-Density Relationship}

Linear regression done in the previous sub-section provides the following model relations between flow and density in the three sites studied.

$$
\begin{aligned}
\text { At Farmgate, } \mathrm{q} & =73.16 \mathrm{k}-19.96 \mathrm{k}^{2} \\
\text { At Shahbag, } \mathrm{q} & =69.49 \mathrm{k}-30.77 \mathrm{k}^{2} \\
\text { At Shukrabad, } \mathrm{q} & =69.73 \mathrm{k}-35.78 \mathrm{k}^{2}
\end{aligned}
$$

The empirical data can be plotted to determine the fitting goodness of data. (figures 7, 8, and 9) 


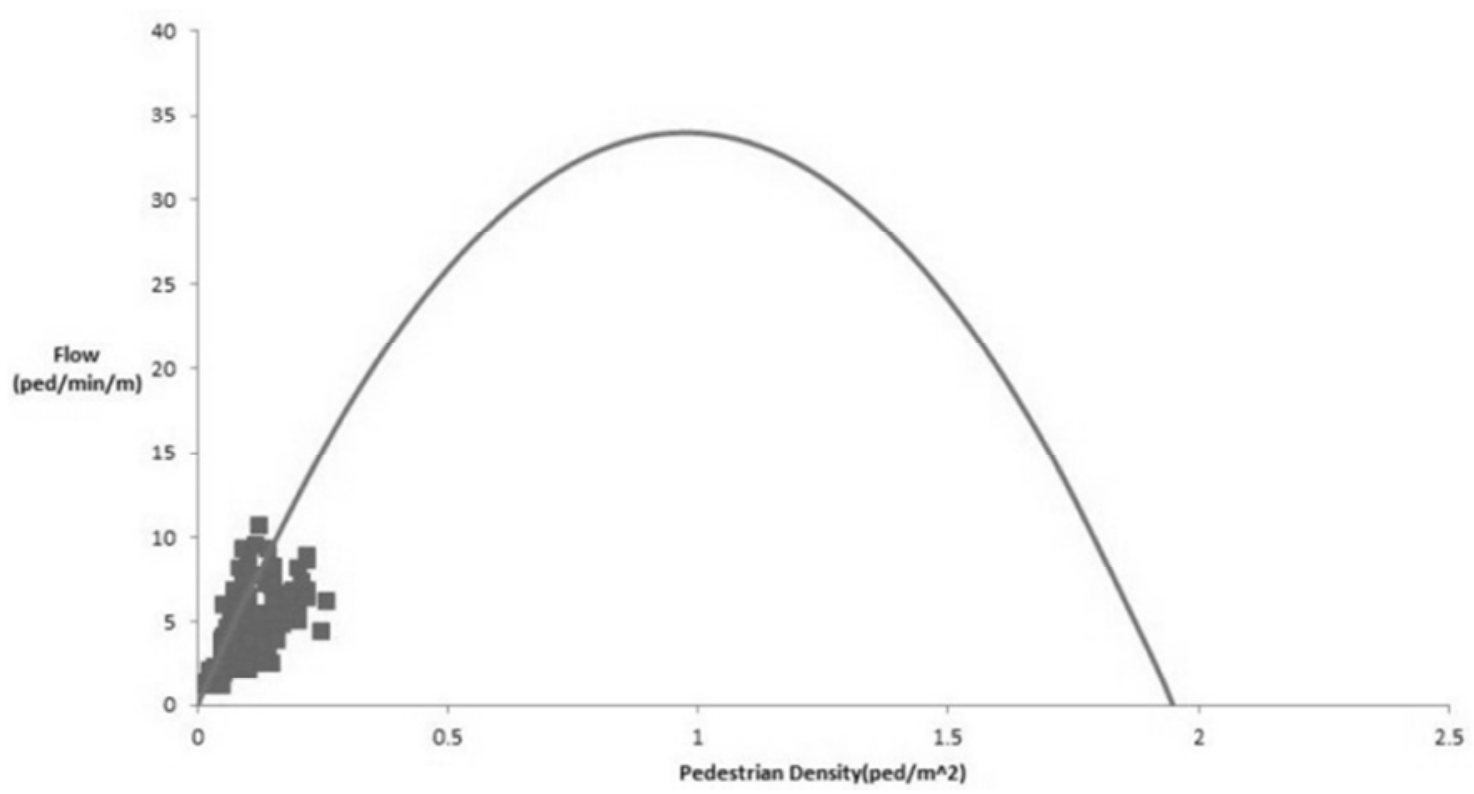

Figure 7. Flow vs. density at Shukrabad.

\subsubsection{Speed-Flow Relationship}

Speed-flow equations are obtained similarly from least square regression of speed-density dataat three locations.

$$
\begin{aligned}
& q=\frac{\mu(73.16-\mu)}{19.96} \\
& q=\frac{\mu(69.48-\mu)}{30.77}
\end{aligned}
$$

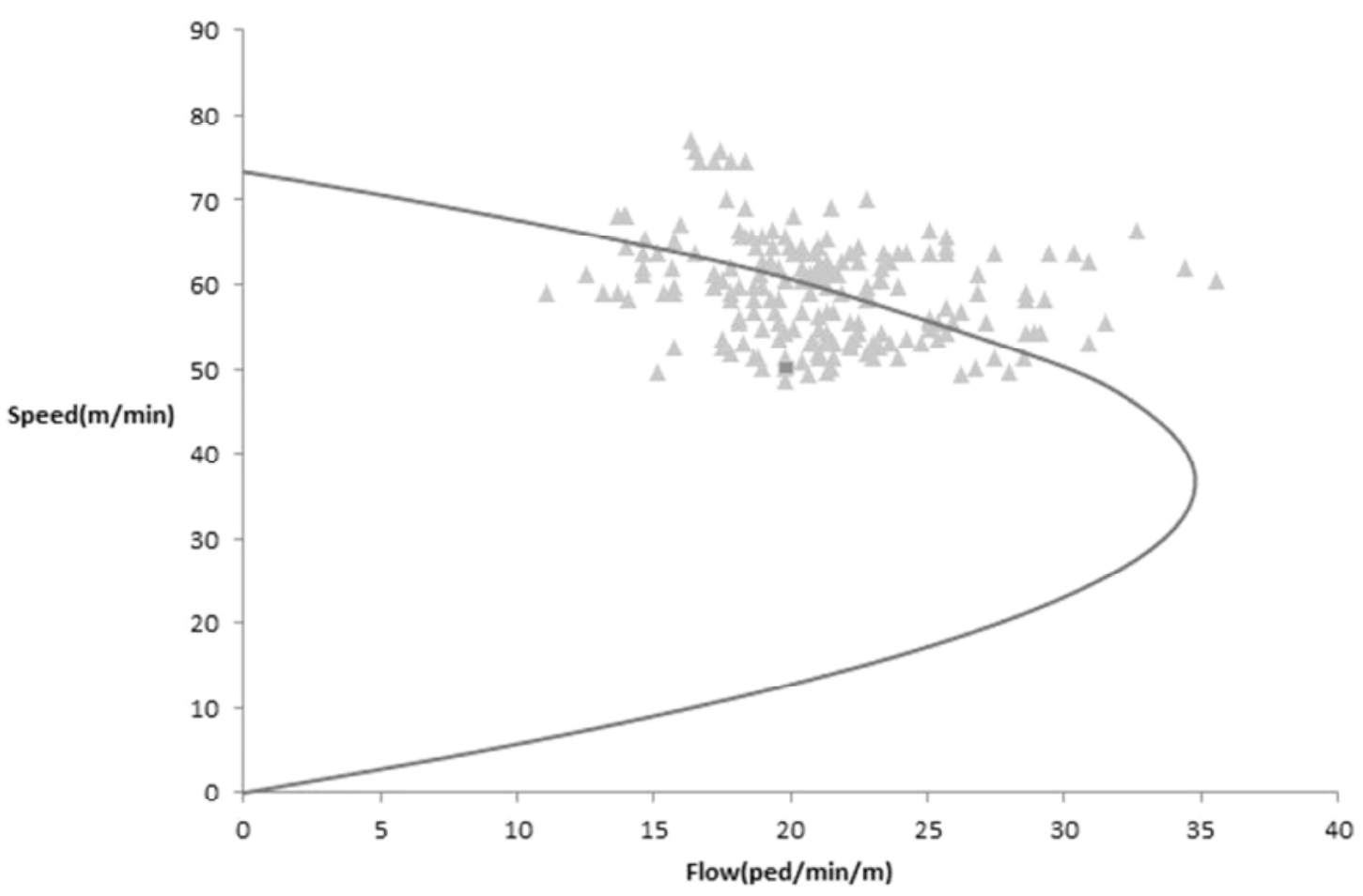

Figure 8. Speed vs. flow at Farmgate.

Similarly, these equations can be used to draw $2^{\text {nd }}$ degree polynomial graphs. The empirical datacan be plotted to determine the fitting goodness of data (figures 10, 11 and 12).

$$
\mathrm{q}=\frac{\mu(69.73-\mu)}{35.78}
$$




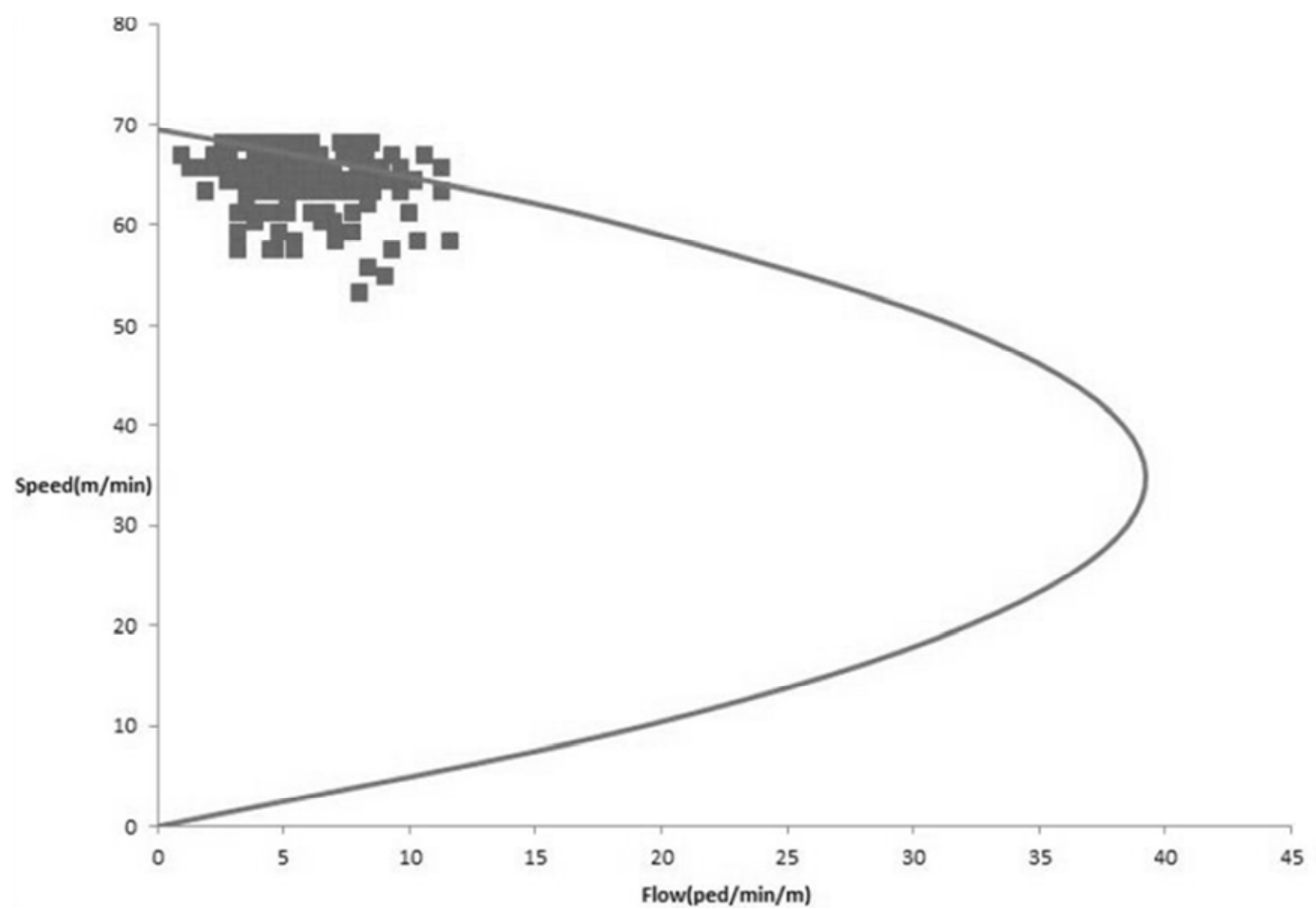

Figure 9. Speed vs Flow at Shahbag.

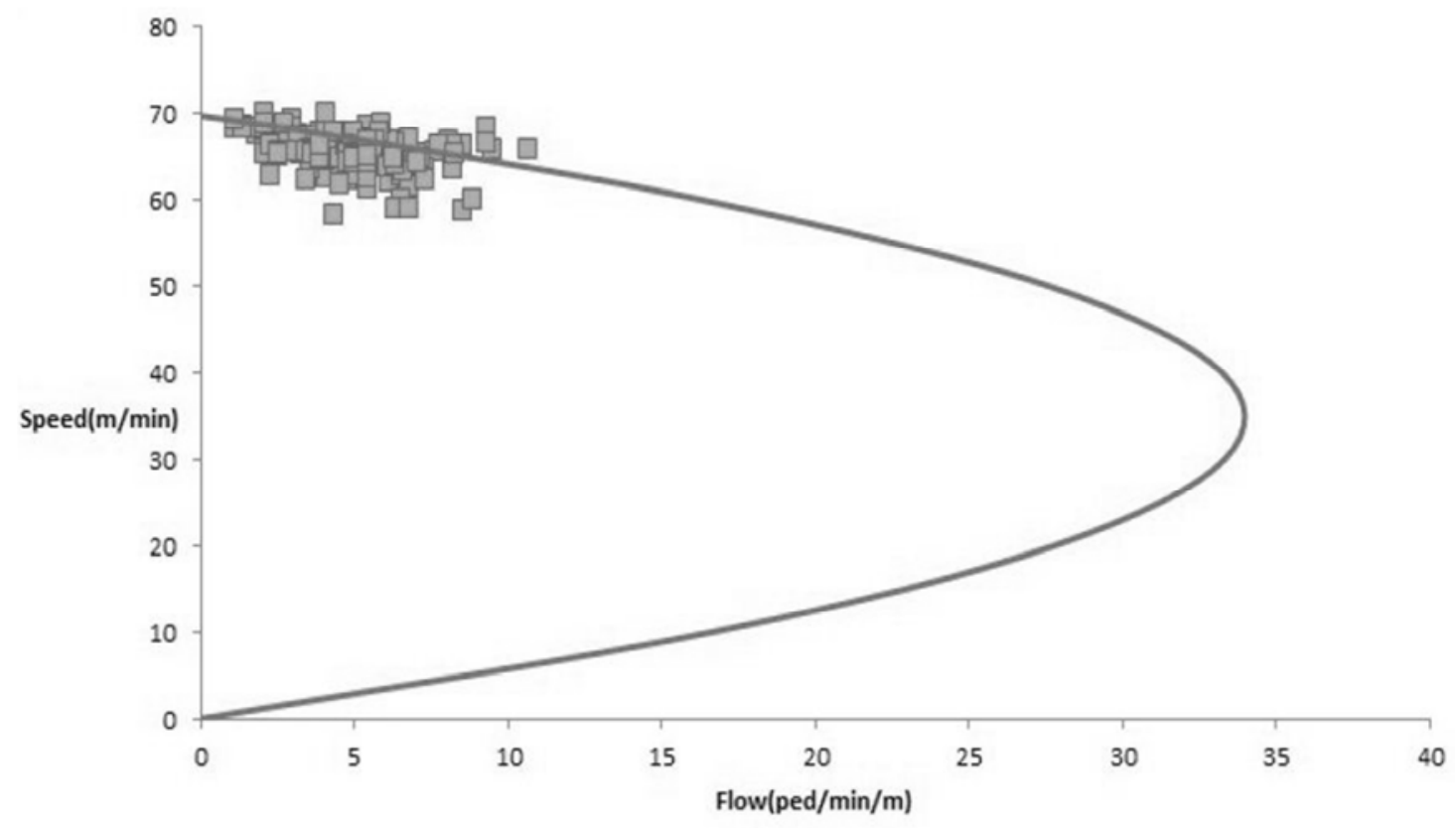

Figure 10. Speed vs Flow at Shukrabad.

\subsection{Pedestrian Flow Relationships}

Tables 1 to 6 summarize the findings of the analysis in tabular form.

Table 1. Pedestrian Density Comparison.

\begin{tabular}{llll}
\hline Location & Type of Area & Maximum observed pedestrian Density $\left(\mathbf{p e d} / \mathbf{m}^{2}\right)$ & Minimum observed pedestrian Density $\left(\mathbf{p e d} / \mathbf{m}^{2}\right)$ \\
\hline Farmgate & Mixed Traffic & 0.78 & 0.18 \\
Shahbag & Mixed Traffic & 0.40 & 0.04 \\
Shukrabad & Mixed Traffic & 0.26 & 0.02 \\
\hline
\end{tabular}


Table 2. Pedestrian Flow Rate Comparison.

\begin{tabular}{llll}
\hline Location & Type of Area & $\begin{array}{l}\text { Maximum flow } \\
\text { rate(ped/m/min) }\end{array}$ & $\begin{array}{l}\text { Minimum flow } \\
\text { rate(ped/m/min) }\end{array}$ \\
\hline Farmgate & Mixed Traffic & 35.58 & 11.08 \\
Shahbag & Mixed Traffic & 11.61 & 0.97 \\
Shukrabad & Mixed Traffic & 10.68 & 1.14 \\
\hline
\end{tabular}

Table 3. Pedestrian Walking Speed Comparison.

\begin{tabular}{llll}
\hline Location & Type of Area & $\begin{array}{l}\text { Maximum } \\
\text { observed walking } \\
\text { speed(m/min) }\end{array}$ & $\begin{array}{l}\text { Minimum } \\
\text { observed walking } \\
\text { speed(m/min) }\end{array}$ \\
\hline Farmgate & Mixed Traffic & 72.32 & 57.52 \\
Shahbag & Mixed Traffic & 68.00 & 53.22 \\
Shukrabad & Mixed Traffic & 69.98 & 58.25 \\
\hline
\end{tabular}

Table 4. Relationships between pedestrian flow characteristics parameters.

\begin{tabular}{llll}
\hline \multirow{2}{*}{ Location } & \multicolumn{1}{c}{ Relationships } \\
\cline { 2 - 4 } & Speed-Density $\boldsymbol{\mu}=\mathbf{f}(\mathbf{k})$ & Flow-Densityq $=\mathbf{f}(\mathbf{k})$ & Speed-Flowq $=\mathbf{f}(\boldsymbol{\mu})$ \\
\hline Farmgate & $\mu=73.16-19.96 \mathrm{k}$ & $\mathrm{q}=73.16 \mathrm{k}-19.96 \mathrm{k}^{2}$ & $\mathrm{q}=\frac{\mu(73.16-\mu)}{19.96}$ \\
Shahbag & $\mu=69.49-30.77 \mathrm{k}$ & $\mathrm{q}=69.49 \mathrm{k}-30.77 \mathrm{k}^{2}$ & $\mathrm{q}=\frac{\mu(69.48-\mu)}{30.77}$ \\
Shukrabad & $\mu=69.73-35.78 \mathrm{k}$ & $\mathrm{q}=69.73 \mathrm{k}-35.78 \mathrm{k}^{2}$ & $\mathrm{q}=\frac{\mu(69.73-\mu)}{35.78}$ \\
\hline
\end{tabular}

Table 5. Pedestrian Flow Characteristic Parameters at different locations.

\begin{tabular}{llllll}
\hline Location & Type of Area & \multirow{2}{*}{ Free flow speed $\boldsymbol{\mu}_{\mathbf{f}}(\mathbf{m} / \mathbf{m i n})$} & $\begin{array}{l}\text { Traffic jam Density } \\
\mathbf{k}_{\mathbf{j}}\left(\mathbf{p e d} / \mathbf{m}^{2}\right)\end{array}$ & Ratio $\psi\left(\mathbf{p e d} . \mathbf{m i n} / \mathbf{m}^{3}\right)$ & $\begin{array}{l}\text { Maximumflow rate } \\
\mathbf{q}_{\mathbf{m}}(\mathbf{p e d} / \mathbf{m} / \mathbf{m i n})\end{array}$ \\
\hline Farmgate & Mixed Traffic & 73.16 & 3.66 & 19.96 & 35.58 \\
Shahbag & Mixed Traffic & 69.49 & 2.26 & 30.77 & 11.61 \\
Shukrabad & Mixed Traffic & 69.73 & 1.95 & 35.78 & 10.68 \\
\hline
\end{tabular}

Table 6. Pedestrian Level of Service(LOS) at three different locations.

\begin{tabular}{llllll}
\hline Location & Type of Area & $\begin{array}{l}\text { Free flow speed } \\
\boldsymbol{\mu}_{\mathrm{f}}(\mathbf{m} / \mathbf{m i n})\end{array}$ & LOS based on speed & $\begin{array}{l}\text { Maximumflow rate } \\
\mathbf{q}(\mathbf{m}(\mathbf{p e d} / \mathbf{m} / \mathbf{m i n})\end{array}$ & $\begin{array}{l}\text { LOS based on flow } \\
\text { rate }\end{array}$ \\
\hline Farmgate & Mixed Traffic & 73.16 & $\mathrm{C}$ & 35.58 & $\mathrm{C}$ \\
Shahbag & Mixed Traffic & 69.49 & $\mathrm{D}$ & 11.61 & $\mathrm{~B}$ \\
Shukrabad & Mixed Traffic & 69.73 & $\mathrm{D}$ & 10.68 & $\mathrm{~B}$ \\
\hline
\end{tabular}

\section{Discussion on Results Found}

1. The speed-density data scattering suggests a negative linear relationship between the two parameters (figure 4, figure 5, figure 6)

2. The flow-density and speed-flow graphs are drawn from parameters found in speed-density relationships. The relationships show a 2nd order polynomial relation. Single regime models calibrated by least square method can't effectively fit the empirical data. However, the models provide 'fair' to 'good' fitting to the empirical data.

3. The traffic jam density at Farmgate was highest (3.6648 $\left.\mathrm{ped} / \mathrm{m}^{2}\right)$ and lowest at Shukrabad $\left(1.9487 \mathrm{ped} / \mathrm{m}^{2}\right)$. This occurred due to the nature of the areas. Farmgate is a very attractive commercial place for shoppers while Shukrabad sidewalk which was under consideration was only used by residents of nearby areas and students.

4. For Farmgate pedestrian trap the increase in flow occurs at 7:00 AM to 8:00 AM, 11:00 AM to 12:00 AM, 12:00 PM to 1:00 PM and 2:00 PM to 3:00 PM. Shahbag experiences spike in pedestrian flow from 8:00 AM to 9:00 AM and 11:00 AM to 2:00 PM. Shukrabad experiences a spike in flow from 2:00 PM to 4:00 PM. The probable cause for this lies in the nature of the study traps themselves. Farmgate is a communication hub especially at the early portion of the day.
Hence the spike in pedestrian flow at 11:00 AM to 1:00 PM rise in flow rate can be attributed to people coming in Farmgate for shopping. While, students coming out of the schools after their break contributes heavily towards the 2:00 to 3:00 PM peak. However, the peak in flow in Farmgate is sporadic because the area itself is always visited by people around the day. Shahbag experiences morning spike due to early commuters as the pedestrian trips in Shahbag are dominated by commuters. Another group of pedestrians that dominate the Shahbag study trap is medical attention seeking people. The late morning to mid-day spike can be attributed to this group of people. As it is more logical that, people will not go to hospitals very early in the day or very late in the day. The morning and noon-afternoon spike in flow rates in Shukrabad can be attributed to school/varsity going and school/varsity leaving students respectively.

5. The maximum flow rate occurs at Farmgate (35.5789 $\mathrm{ped} / \mathrm{m} / \mathrm{min}$ ) while the minimum flow occurs at Shahbag ( $0.9677 \mathrm{ped} / \mathrm{m} / \mathrm{min})$. The maximum value occurs at Farmgate due to its' high attraction for shoppers while the lowest value occurs at Shahbag during the late part of the noon where there are very few commuters as well as medical attention seeking people.

6. The maximum free flow walking speed instance occurred in Farmgate $(73.161 \mathrm{~m} / \mathrm{min})$ while minimum free flow walking speed instance was observed also in Shahbag 
$(69.488 \mathrm{~m} / \mathrm{min})$. This expresses a certain aspect of human psychology. People face the most friction in Farmgate study trap. Hence they try to cover this length as fast as possible. Unless, people are actually stopped by overwhelming number of other pedestrians, they will try to walk past the sidewalk with highest friction as fast as possible.

7. The speed-density curves in each location shows fairly good distribution. However, this is expected since the best fit line was drawn with the help of Microsoft Excel.

8 . The free flow speed ranges from $69-74 \mathrm{~m} / \mathrm{min}$ which is much less than that of USA, Germany but similar to that of China, Saudi Arabia, India, Kuwait, Philippines, Thailand and Japan. This is expected since for differences in cultural values people in the western countries tend to walk faster.

9. Traffic jam density in each of these three locations were much less than that of China, Thailand and Singapore and similar to that of India and Saudi Arabia

10. The US Highway Capacity Manual standard produced a table showing the relationships between spaces, average speed and flow rates at different levels of service. This table considers both walking speed and flow rates as basis for LOS classification. However, for the type of pedestrian traps used in this study a more realistic approach more denoting LOS will be on the basis of flow rate. On this basis Farmgate has a LOS C and Shukrabad and Shahbag both have a LOS B. According to $\mathrm{HCM}$ at LOS $\mathrm{C}$ found at Farmgate space is sufficient for normal walking speeds, and for bypassing other pedestrians in primarily unidirectional streams. Reversedirection or crossing movements can cause minor conflicts, and speeds are somewhat lower [8] [9]. Similarly, at Shahbag and Shukrabad (at LOS B) there is sufficient area for pedestrians to select walking speeds freely to bypass other pedestrians, and to avoid crossing conflicts. At this level, pedestrians begin to be aware of other pedestrians, and to response to their presence when electing a walking path.

\section{Conclusions}

In Bangladesh, with a low level of motorization and greater urban poverty, the role of walking is quite significant. However, pedestrians are highly vulnerable as well. The walking speed, flow and density of pedestrians are of prime importance in a study for design and provision of pedestrian facilities. Large proportions of sidewalk users' need the provision of walk facilities such as exclusive walkways or footpaths on the road for their safe movement. Pedestrians walk differently on different types of walking facilities [12] [14].

Pedestrian characteristics are mainly represented in this study through three parameters, speed, density and flow rate. The speed-density has linear relationship with negative slope. Therefore, as the density increases the speed of the pedestrian decreases. However, this might not always be applicable to pedestrian movements. The relationships among the variables are expressed through mathematical equations. These equations can be used for macroscopic modeling purposes. It indicates the maximum capacity of the sidewalk as well as provides current level of service for pedestrian movements. The study has shed some light on the walking speeds of pedestrians in Dhaka City compared to that of other countries and cities. The average free-flow speed from three locations is about $1.18 \mathrm{~m} / \mathrm{s}$. This might help create an idea about the rate at which pedestrians might clear out from facilities.

Contrary to popular belief increase in density might not always indicate slowing down of pedestrians. The pedestrians will try to escape side frictions as fast as they can upon recognizing it. Only after being obstructed by high density, people will be forced to slow down their walking speed. People usually walk much slower in groups and people of female sex tend to walk slower than their male counterpart. The sidewalk on Farmgate to a large extent was encroached by street hawkers or vendors increasing roadside friction. While the sidewalk on Shukrabaad was free from obstructions allowing pedestrians to move freely. Roadside developments and constructions encourage people to move faster to escape the obstructions while street hawkers attract the pedestrians causing them to move slower or come to a halt altogether. Width of the sidewalks, pedestrian density, side frictions, presence of walking partners, age and gender all these factors together contribute towards determining the walking speed of pedestrians. Each of these factors have an effect on the pedestrian flow characteristics and their overall effects need to be considered before reaching specific conclusions for designing pedestrian facilities.

\section{References}

[1] Al-Masaeid, H. R., Al-Suleiman, T. I. and Nelson, D. C (1993) "Pedestrian Speed Flow Relationship for Central Business Areas in Developing Countries", Transportation Research Record 1396, National Research Council, Washington, 69-74.

[2] BBS (Bangladesh Bureau of Statistics).: Bangladesh Population Sensus-2001: Planning Division, Ministry of Planning, Government of the People's Republic of Bangladesh, 2007.

[3] WHO Report, (2001) "Statistical annex Geneva world health organization, 2001",

http://www.who.int/whr/2001/annex/en/index.html.

[4] Fruin, John J (1971) "Pedestrian planning and design", Elevator World Inc., Ala.

[5] Fruin, J. J (1971a): Pedestrian and Planning Design, Metropolitan Association of Urban Designers and Environmental Planners Inc., New York.

[6] Fruin, JJ., (1970) "Designing for pedestrians: A level of service concept" Ph.D. Thesis, Polytechnic Institute of Brooklyn, pp: 151.

[7] Gerilla, G. P. (1995) "Proposed Level of Service Standards for Walkways in Metro Manila," Journal of the Eastern Asia Society for Transportation Studies, 1(3), Autumn 1995.

[8] Hoogendoorn, S. P, and Daamen, W., (2005a) "Pedestrian Behavior at Bottlenecks," Transportation Science, Informs, $39(2), 147-159$. 
[9] Hoque M. M. \& Mahmud S. M. S. (2010), "Promoting vulnerable road users' safety towards safe and equitable communities in Bangladesh", InjPrev 2010; 16: A152 doi: 10.1136/ip.2010. 029215.545. British Medical Journal (BMJ Journals).

[10] Hughes, R. L. (2002) "A Continuum Theory for the Flow of Pedestrians.” Transportation Research Part-B, 36(6), 507-535.

[11] Lam, W. H. K. and Cheung, C., (2000) "Pedestrian Speed/Flow Relationships for Walking Facilities in Hong Kong, Journal of Transportation Engineering, ASCE, 126 (4), 343-349.

[12] Lam, W. H. K., Morrall, J. F., and Ho, H., (1995), "Pedestrian Flow Characteristics in Hong Kong', Transportation Research Record 1487, TRB, National Research Council, 56-62.

[13] Laxman, K. Rastogi, R., and Chandra, S. (2010) "Pedestrian Flow Characteristics in Mixed Traffic Conditions." J. Urban Plann. Dev., 10.1061/(ASCE)0733-9488(2010)136: 1(23), 2333.72.

[14] Navin, F. P. D., and Wheeler R. J. (1969), "Pedestrian flow characteristics", Traffic Engineering and Control, 39(9), 3036.

\section{Biography}

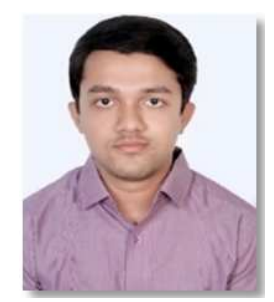

A. M. Tahsin Emtenan is working as a Lecturer of Civil Engineering Department in Port City International University. $\mathrm{He}$ graduated from Bangladesh University of Engineering and Technology with a B. Sc. degree in Civil Engineering in 2016. His research interest focuses on Transportation

Engineering.

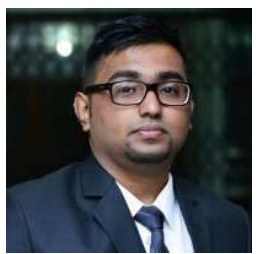

Showkat Ibne Shahid is working as an Assistant Engineer in Bangladesh Water Development Board. He graduated from Bangladesh University of Engineering and Technology with a B. Sc. degree in Civil Engineering in 2016. His research interest focuses on Transportation Engineering. 